\title{
Análisis de los problemas de probabilidad propuestos en las pruebas de acceso a la Universidad en Andalucía
}

\author{
María del Mar López-Martín, Universidad de Granada (España) \\ José Miguel Contreras, Universidad de Granada (España) \\ Magdalena Carretero, IES Miguel de Cervantes, Granada (España) \\ Luis Serrano, Universidad de Granada (España)
}

Recibido el 23 de Marzo de 2015; aceptado el 30 de Noviembre de 2015

\begin{abstract}
Análisis de los problemas de probabilidad propuestos en las pruebas de acceso a la Universidad en Andalucía

Resumen

El objetivo de este estudio fue, analizar el contenido de los problemas de probabilidad incluidos en las pruebas de Selectividad del Distrito Andaluz en la especialidad de Bachillerato de Ciencias Sociales. Se han analizado las pruebas realizadas desde 2003 a 2014. Los problemas de probabilidad se han resuelto y mediante un análisis semiótico se ha identificado los experimentos incluidos en el enunciado, el tipo de probabilidad pedida (simple, compuesta y condicional), los teoremas requeridos en su solución y los contextos utilizados en el problema. Un estudio descriptivo de la frecuencia de estas variables permite obtener conclusiones sobre la presencia y la dificultad de los mismos. Estos resultados pueden servir para la elaboración de pruebas futuras y preparar a los estudiantes que tienen que enfrentarse a las mismas.
\end{abstract}

Palabras clave. Probabilidad; pruebas de acceso; análisis de problemas; evaluación.

\begin{abstract}
Análise de problemas de probabilidade propostos nos exames de admissão para a universidade em Andalucía

Resumo

O objetivo deste estudo foi analisar o conteúdo dos problemas de probabilidade proposto nos exames de admissão para a universidade em Andalucía na especialidade de Bacharelado em Ciências Sociais. Foram analisados os testes propostos de 2003 a 2014. Os problemas de probabilidade propostos foram resolvidos e através de uma análise semiótica se identificou as experiências incluidas no título, o tipo de probabilidade solicitada (simples, composta e condicional), os teoremas necessários em sua solução e os contextosutilizados em el problema. Um estudo estatístico elementar da frequência de cada um dos objetos nos três anos permite conclusões sobre a presença e da dificuldade do mesmo. Estes resultados podem ser utilizados para melhorar estes testes e preparar os alunos que têm de lidar com eles.
\end{abstract}

Palavras chave. Possibilidade; exames de admissão; análise de problemas; avaliação

Para citar: Lopéz-Martín, M.M., Contreras, J.M., Carretero, M. \& Serrano, L. (2016). Análisis de los problemas de probabilidad propuestos en las pruebas de acceso a la Universidad en Andalucía. Avances de Investigación en Educación Matemática, 9, 65 - 84.

(C) Sociedad Española de Investigación en Educación Matemática (SEIEM). www.seiem.es 
Analysis of probability problems proposed in the entrance to university tests in Andalucía

\section{Abstract}

The aim of this study was to analyze the content of the probability problems included for the university entrance tests in Andalucia (Spain) in the specialty of Social Sciences. We analyzed the tests proposed from 2003 to 2014. The probability problems have been solved and through a semiotic analysis we identified the experiments included, the type of probability requested (simple, compound and conditional), the theorems required in its solution and the contexts used in the problem. An elementary statistical study of the frequency of each object served to conclude about the presence and the difficulty of the same. These results can be used to improve these tests and prepare the students to deal with them.

Key words. Probability; university entrance tests in Andalucía; analysis of problems; evaluation.

Analyse des problèmes de probabilité proposée dans les examens d'entrée à l'université en Andalousie

\section{Résumé}

Le but de cette étude est d'analyser le contenu des problèmes de probabilités proposées dans les sélectivités tests en Andalousie dans la spécialité de baccalauréat en sciences sociales. Nous avons analysé les tests proposés de 2003 à 2014. Les problèmes de probabilité proposées ont été résolus et à travers une analyse sémiotique on a identifié les expériences incluses, le type de probabilité demandé (simple, composé et conditionnelle), les théorèmes nécessaires dans sa solution et les contextes utilisés dans le problème. Une étude statistique élémentaire de la fréquence de chacun des objets permet des conclusions sur leur présence et la difficulté des problèmes. Ces résultats peuvent être utilisés pour améliorer ces tests et de préparer les étudiants qui ont à traiter avec eux.

Paroles clés. Chance; examens d'entrée; l'analyse des problèmes ; l'évaluation.

\section{Introducción}

Aunque el estudio de la estadística y la probabilidad está recogido desde hace años en los planes de estudios de todas las etapas educativas, no se le ha llegado a dar la importancia que tiene en realidad. No obstante, en los últimos años se ha observado un cambio de esta situación. Un ejemplo de ello lo encontramos en el contexto de las pruebas PISA (OCDE, 2009), al reconocer que es necesario que los estudiantes desarrollen capacidades para enfrentarse en su vida diaria y profesional a la toma de decisiones, desde una perspectiva matemática y científica.

Nuestro trabajo se enfoca en analizar las variables que determinan los problemas de probabilidad incluidos en las Pruebas de Acceso a la Universidad (en adelante PAU) de la Comunidad Autónoma de Andalucía. Consideramos el término problema en sentido muy amplio, como cualquier ejercicio o tarea que el alumno ha de resolver y en particular, los ítems que se les plantean en las PAU.

Dentro del ámbito de la enseñanza matemática el estudio de las pruebas de evaluación juega un papel importante, ya que el análisis de las soluciones finales, estrategias, argumentos, símbolos usados, etc., permiten indirectamente evaluar el aprendizaje de los estudiantes (Godino, 1996). Consecuentemente, se deduce la importancia de analizar las pruebas de evaluación, para asegurar que haya una correspondencia entre el contenido pretendido en las orientaciones curriculares para un tema y el evaluado. 


\section{Fundamentación teórica}

En primer lugar se presentan los fundamentos de nuestro trabajo que consisten en un análisis del contenido de probabilidad en el currículo de Bachillerato, la normativa de las PAU y algunos antecedentes de nuestra investigación.

\subsection{La probabilidad en el currículo de Bachillerato}

La necesidad de formación en probabilidad para el alumnado ha sido recogida en las orientaciones curriculares españolas, que contemplan esta formación en Educación Primaria, Educación Secundaria y Bachillerato (Batanero, Arteaga \& Gea, 2011; Batanero, Gea, Arteaga \& Contreras, 2014).

Aunque el estudio de la probabilidad se trata en los diferentes niveles educativos, es en Bachillerato donde cobra mayor importancia. Este tema ha sido incluido en este nivel educativo tanto en la asignatura Matemática I (Ciencias y Tecnología) como en las Matemática Aplicadas a las Ciencias Sociales I y II (Ciencias Sociales y Humanidades), donde se fijaron los siguientes contenidos (MEC, 2007):

- Matemáticas I: Estudio de la probabilidad compuesta, condicionada, total y a posteriori. Distribuciones binomial y normal como herramienta para asignar probabilidades a sucesos (MEC, 2007, p. 45449).

- Matemáticas Aplicadas a las Ciencias Sociales I: Asignación de probabilidades a sucesos. Distribuciones de probabilidad binomial y normal (MEC, 2007, p. 45475).

- Matemáticas Aplicadas a las Ciencias Sociales II: Profundización en los conceptos de probabilidades a priori y a posteriori, probabilidad compuesta, condicionada y total. Teorema de Bayes. Implicaciones prácticas de los teoremas: Central del límite, de aproximación de la Binomial a la Normal y Ley de los Grandes Números. Problemas relacionados con la elección de las muestras. Condiciones de representatividad. (MEC, 2007, p. 45476).

Tabla 1. Contenidos de Probabilidad en Bachillerato.

\begin{tabular}{|c|c|c|c|}
\hline \multirow[t]{2}{*}{ Contenidos } & \multirow{2}{*}{$\begin{array}{c}\text { Ciencias y Tecnología } \\
1^{\mathrm{o}} \text { curso } \\
\end{array}$} & \multicolumn{2}{|c|}{ Ciencias Sociales } \\
\hline & & $1^{\circ}$ curso & $2^{\circ}$ curso \\
\hline $\begin{array}{l}\text { Probabilidad Simple, asignación de } \\
\text { probabilidades }\end{array}$ & & $\mathrm{X}$ & \\
\hline $\begin{array}{l}\text { Probabilidad compuesta, condicionada, total } \\
\text { y a posteriori }\end{array}$ & $\mathrm{X}$ & & $\mathrm{X}$ \\
\hline Teorema de Bayes & $\mathrm{X}$ & & $\mathrm{X}$ \\
\hline Distribuciones Binomial y Normal & $\mathrm{X}$ & $\mathrm{X}$ & \\
\hline $\begin{array}{l}\text { Teorema Central del Límite, aproximación } \\
\text { de la Binomial a la Normal }\end{array}$ & & & $X$ \\
\hline $\begin{array}{l}\text { Ley de los Grandes Números. Muestreo } \\
\text { representatividad }\end{array}$ & & & $X$ \\
\hline
\end{tabular}

Tal y como muestra la Tabla 1, los contenidos probabilísticos descritos en el primer curso de la modalidad de Ciencias y Tecnología son más amplios que en primero de la especialidad de Ciencias Sociales, ya que además de repasar la probabilidad simple, la probabilidad compuesta y condicional introducen el Teorema 
de Bayes. Sin embargo, cabe destacar que el estudio de probabilidad es más completo en la modalidad de Ciencias Sociales debido a que el Bloque de Estadística y Probabilidad, dentro de la modalidad de Ciencias y Tecnología, solamente está contenido en la estructura de primero de Bachillerato.

En las nuevas orientaciones curriculares (MECD, 2015), donde se establecen únicamente tres modalidades de bachillerato (Artes; Ciencias; y Humanidades y Ciencias Sociales), se suprimen los contenidos de probabilidad en la rama de Ciencias y se amplían en la modalidad de Humanidades y Ciencias Sociales tal y como queda reflejado a continuación:

- Matemáticas Aplicadas a las Ciencias Sociales I: Sucesos. Asignación de probabilidades a sucesos mediante la regla de Laplace y a partir de su frecuencia relativa. Axiomática de Kolmogorov. Aplicación de la combinatoria al cálculo de probabilidades. Experimentos simples y compuestos. Probabilidad condicionada. Dependencia e independencia de sucesos (MECD, 2015, p. 385).

- Matemáticas Aplicadas a las Ciencias Sociales II: Profundización en la Teoría de la Probabilidad. Axiomática de Kolmogorov. Asignación de probabilidades a sucesos mediante la regla de Laplace y a partir de su frecuencia relativa. Experimentos simples y compuestos. Probabilidad condicionada. Dependencia e independencia de sucesos. Teoremas de la probabilidad total y de Bayes. Probabilidades iniciales y finales y verosimilitud de un suceso (MECD, 2015, p. 389).

\subsection{Las pruebas de acceso a la universidad (PAU)}

Las actuales pruebas de acceso a la universidad están regidas por el Real Decreto 1892/2008, de 14 de noviembre, por el que se regulan las condiciones para el acceso a las enseñanzas universitarias oficiales de grado y los procedimientos de admisión a las universidades públicas españolas (MP, 2008). A su vez este decreto se basa en la Ley Orgánica de Educación, que exige, en su artículo 38, la superación de una prueba de madurez que permita valorar los conocimientos y la capacidad de los estudiantes para iniciar sus estudios universitarios.

La prueba de Matemáticas Aplicadas a las Ciencias Sociales II ha constado hasta la fecha de dos opciones (opción A y opción B) donde el estudiante, bajo su parecer, tenía posibilidad de elegir únicamente una de las opciones, sin poder entremezclar los ejercicios que componen cada opción. Tanto la opción $\mathrm{A}$, como la opción $\mathrm{B}$, están divididas en tres tipologías de ejercicios. El primer ejercicio pertenece al Bloque de contenidos de Álgebra y el segundo al Bloque de contenidos de Análisis. Finalmente, los ejercicios tercero y cuarto corresponden al Bloque de Probabilidad y Estadística; más concretamente, a probabilidad e inferencia estadística. Aunque en las últimas ediciones, cada uno de los ejercicios que forman la prueba tuvieron asignada una puntuación máxima de 2.5 puntos, no es hasta el curso 2009/2010 cuando se estableció este criterio. Anteriormente, se evaluaba sobre 4 puntos los dos ejercicios pertenecientes al bloque de Probabilidad y Estadística dándole mayor protagonismo a los bloques de Álgebra y Análisis.

En lo que se refiere a la probabilidad nuestro análisis se restringe al siguiente contenido "Profundización en los conceptos de probabilidades a priori y a posteriori, probabilidad compuesta, condicionada y total. Teorema de Bayes" al cual se ha dedicado un ejercicio específico en cada prueba, cubriendo otro ejercicio el resto del contenido de probabilidad y estadística. 


\subsection{Antecedentes}

Entre los estudios realizados sobre otras pruebas de evaluación encontramos algunos trabajos en los que se ha analizado el marco teórico en que se basan dichas pruebas. Un ejemplo de ello es el trabajo de Rico (2006) en el que se relaciona el marco teórico de las pruebas de matemáticas PISA del año 2003 con la resolución de problemas desde el punto de vista curricular, a través del concepto de competencia matemática y el modelo funcional sobre el aprendizaje de las matemáticas. Dicho modelo analiza el patrón que se sigue a la hora de resolver una tarea matemática: campos de actuación (fenómenos, situaciones o contextos y problema que plantea la tarea); herramientas o matemáticas puestas en juego para resolverla (estructuras conceptuales y procedimientos) y procesos cognitivos que se movilizan para su ejecución (pensar y razonar, comunicar, justificar, representar, modelizar, plantear y resolver problemas).

Por otro lado, Caraballo (2010) analiza la competencia matemática de 173 ítems incluidos en las pruebas de diagnóstico correspondientes al segundo curso de Educación Secundaria Obligatoria de cinco comunidades autónomas durante el curso académico 2008-2009. El objetivo principal de su investigación fue estimar la correspondencia entre los instrumentos utilizados por las comunidades autónomas para confeccionar estas pruebas y el modelo PISA. Las variables analizadas en su trabajo fueron: el contenido matemático (cantidad, espacio y forma, cambio y relaciones e incertidumbre); el contexto o situación (personal, público, educativo y profesional y científico); el nivel de complejidad y los procesos que se deben utilizar para realizar una tarea matemática. La autora analizó también la forma de dar la información a los estudiantes. Sus resultados indican un desequilibrio en la distribución de los ítems respecto al contexto y al contenido; también observa que la representación gráfica o simbólica es la forma más frecuente de presentar la información a los estudiantes.

Castellanos (2013) centra su investigación en el estudio de las tablas y gráficos estadísticos en las pruebas SABER de Colombia para estudiantes de último grado del ciclo de educación básica en los años 2003, 2006 y 2009. Las variables analizadas son: tipo de gráfico o tabla utilizado (como por ejemplo el diagrama de sectores o el pictograma); competencia evaluada (razonamiento, resolución o comunicación); nivel de lectura de gráficos (tipo de dato que debe obtener del gráfico); actividad solicitada (interpretar una representación, organizar y representar datos) y los niveles de complejidad semiótica del gráfico -entre los descritos por Arteaga (2011)-. Del estudio realizado concluye que el gráfico más frecuentemente usado es el diagrama de barra; los ítems destacan la competencia en comunicación y el nivel de lectura entre los datos (que requiere la realización de comparaciones y cálculos). Usualmente estos gráficos y tablas representan una distribución de datos, es decir, corresponderían a un nivel avanzado de complejidad semiótica según Arteaga (2011).

Recientemente, Mingorance (2014) analizó los gráficos y tablas estadísticas de las pruebas de diagnóstico andaluzas obligatorias para los niños de 10 años observando una alta frecuencia de los gráficos de barras frente a la escasez de todos los recomendados en el currículo. Según la actividad que se solicita al estudiante (organizar, comprender e interpretar la información) concluye que el nivel de competencia requerido para resolver los problemas es bajo; los contextos preferentes son personales y sociales. Generalmente las actividades están más centradas en la 
lectura, traducción o finalización del gráfico que en su interpretación o construcción completa.

\section{Metodología}

La investigación llevada a cabo es de tipo cualitativa ya que se basa en el análisis de contenido. Esta técnica supone que un texto puede dividirse en unidades que pueden clasificarse en un número reducido de categorías en función de variables subyacentes, y que permiten realizar inferencias sobre su contenido (Krippendorff, 1997).

Según Bisquerra (1989), el proceso de investigación que se ha desarrollado es inductivo, pues se parte de casos particulares (ejemplo de pruebas PAU) con el fin de obtener generalizaciones a partir de estas observaciones. De acuerdo a dicho autor, podemos clasificar la investigación realizada como aplicada, puesto que tratamos de encontrar resultados aplicables en educación, y exploratoria, ya que está encaminada a obtener conocimientos sobre las características de la evaluación realizada a los estudiantes, pero no se consideran hipótesis de partida.

\subsection{Muestra de problemas analizados}

La muestra utilizada está formada por todos los problemas propuestos en las pruebas planteadas en Matemáticas Aplicadas a las Ciencias Sociales en Andalucía durante los años 2003 a 2014, en total 12 años. En cada uno de estos años se revisaron las 6 pruebas disponibles en las convocatorias de Junio y Septiembre, en cada una de las cuales se propuso un problema de probabilidad. Por tanto, la muestra se compone de un total de 144 problemas.

De cada uno de los ítems se ha analizado el contenido matemático, determinado a partir del análisis del enunciado y de una posible solución de cada apartado determinado por los investigadores. Se utilizó para ello el análisis semiótico (Godino, Batanero \& Font, 2007), determinando los objetos matemáticos implícitos en dicha resolución.

Es importante señalar que la muestra con la que se está trabajando es de tipo intencional, lo que es propio de la metodología cualitativa. Por tanto, no se pretende extrapolar los resultados a otras pruebas diferentes a las analizadas.

\subsection{Variables consideradas}

Dentro de los objetivos mínimos de probabilidad exigidos al estudiante en la prueba PAU (Consejería Educación, 2008) encontramos que: El estudiante deberá comprender y manejar el lenguaje probabilístico. Ha de ser capaz de construir el espacio muestral asociado a un experimento aleatorio simple, describiendo los sucesos asociados a él y realizar operaciones con ellos como la unión y la intersección de sucesos; asimismo debe ser capaz de formar el espacio muestral asociado a un experimento aleatorio compuesto y restringirlo cuando se da una condición dada. Determinará la probabilidad de un suceso a partir de la regla de Laplace, cuando los sucesos sean equiprobables, o aplicando las propiedades de experimentos compuestos y de la realización o no de dos o tres sucesos independientes. Dominará el teorema de 
la probabilidad total y el teorema de Bayes, diferenciado entre sucesos a priori y a posteriori, aplicando los cálculos correctamente.

Considerando los objetivos mencionados y los contenidos matemáticos, se han deducido las siguientes variables, las cuales han sido analizadas en todos los problemas que componen la muestra:

- V1: Tipos de experimentos incluidos en el item. Puesto que trabajamos con problemas de probabilidad, siempre hay uno o varios experimentos aleatorios descritos explícitamente o bien que se sobreentienden implícitamente en el enunciado. Ortiz (2002) analiza en su estudio el espacio muestral asociado a los problemas presentados en los libros de texto y los diferencia entre espacios muestrales simples o compuestos; argumenta que esta variable influye en la dificultad del problema. Debido a esto, se ha considerado de interés la variable tipo de experimento aleatorio que aparece implícita o explícitamente en el enunciado del problema.

- V2. Tipos de probabilidades pedidas. Se pueden solicitar probabilidades simples, compuestas o condicionales, cada una de las cuáles tiene diferente dificultad y son confundidas por los estudiantes, como se muestra, por ejemplo en Díaz (2007) y Contreras (2011).

- V3. Teorema o propiedades utilizadas. Debido a que el uso del teorema de la probabilidad total o teorema de Bayes muestra ciertas dificultades de razonamiento en los estudiantes (Díaz, Ortiz \& Serrano, 2008), se ha analizado si la resolución del ejercicio planteado requiere el empleo de tales herramientas.

- V4. Contexto del ítem. Tenemos en cuenta los contextos considerados en las pruebas PISA de evaluación organizados por la Organización para la Cooperación y el Desarrollo Económico (OECD). Estas pruebas están orientadas a evaluar la competencia matemática; más concretamente, evalúan "la capacidad de formular, emplear e interpretar cuestiones matemáticas en diferente tipo de contextos" (MCD, 2013, p. 11) mediante la resolución de tareas relacionadas con la vida real (ME, 2009, MCD, 2013). Los contextos son personal, profesional, social y científico. En aquellos casos donde el problema no se ha descrito sobre alguno de los contextos mencionados se considera que el problema no tiene contexto.

\section{Resultados}

Una vez se eligieron las variables a analizar se identificaron las posibles categorías para cada una de estas variables y para cada problema, codificando los datos para su posterior tratamiento estadístico con el software Excel. En lo que sigue se presentan los resultados describiendo las categorías de cada una de las variables junto con un ejemplo que las clarifique y además, se presenta la distribución, globalmente y por año, de las categorías en la muestra de problemas.

\subsection{Tipos de experimentos incluidos en el ítem}

En el cálculo de una probabilidad siempre es considerado un cierto experimento aleatorio junto con el espacio muestral correspondiente. Como consecuencia, el 
estudiante necesita identificar el experimento que viene asociado al problema que debe realizar. Siguiendo las sugerencias de Ortiz (2002), cada uno de los elementos muestrales de los problemas analizados se ha clasificado como experimento simple o compuesto. Para ello se ha considerado que un experimento simple es aquel experimento aleatorio que no puede descomponerse en otros experimentos, mientras que un experimento compuesto es aquel experimento aleatorio compuesto por al menos dos experimentos aleatorios simples. Por ejemplo, si se considera el experimento de lanzar una moneda una única vez se estaría ante un experimento aleatorio simple, pero si realizamos el experimento de lanzar una moneda dos veces, de forma consecutiva, entonces el experimento es compuesto por estar formado por dos experimentos simples. Un ejemplo de experimento simple aparece en el ítem P2B propuesto el año 2008:

Ítem P2B (2008). Se consideran los sucesos $A$ y $B$.

a. Exprese, utilizando las operaciones con sucesos, los siguientes sucesos: 1. Que no ocurra ninguno de los dos. 2. Que ocurra al menos uno de los dos. 3. Que ocurra $B$, pero que no ocurra $A$.

b. Sabiendo que $P(A)=0$ '5, $P(B)=0$ '5 y $P(A \mid B)=0$ '3, halle $P(A \cup B)$.

En este caso el experimento aleatorio es simple ya que no se hace referencia a probabilidad conjunta y es posible encontrar la probabilidad de la unión y la probabilidad condicional. Por otro lado, un ejemplo de experimento aleatorio compuesto es el ítem P1A propuesto en el año 2003 cuyo enunciado es el siguiente:

Ítem P1A (2003). El 55 \% de la población española son mujeres, de las cuales un $23 \%$ usa el coche para ir al trabajo. Se sabe que la probabilidad de que una persona, sea hombre o mujer, vaya al trabajo en coche es 0 ' 52 .

a. Elegido un hombre, al azar, ¿cuál es la probabilidad de que utilice el coche para desplazarse al trabajo?

b. Si se elige una persona, al azar, y resulta que no usa el coche para ir al trabajo, calcule la probabilidad de que sea una mujer.

El experimento es de tipo compuesto pues se plantea un experimento determinado por dos experimentos simples y diferentes, E1: Elegir una persona al azar y observar su género (ser hombre o mujer) y E2: Una vez seleccionada la persona se le pregunta cómo se desplaza al trabajo (usa el coche para ir al trabajo o no). Este tipo de experimentos introduce una mayor complejidad al problema ya que es necesario detectar la posible dependencia o independencia existente entre los sucesos

Realizado el estudio sobre todos los ítems se observa que no existen diferencias significativas entre el número de problemas propuestos en el que el experimento es de tipo simple o compuesto, ya que aproximadamente el $56 \%$ de ellos son simples y el resto son compuestos.

Si se analiza esta variable en la muestra por año (Figura 1) se observa una gran variabilidad, desde algunos años como 2003 en que prácticamente todos los experimentos propuestos fueron compuestos hasta otros como 2013 en que ocurrió lo contrario. De los doce años estudiados se observa que en ocho de ellos el número de experimentos compuestos fue mayor que el de simple. Debido a la complejidad que conlleva trabajar con experimentos compuestos indica que la dificultad de los problemas propuestos no es la misma todos los años. 


\subsection{Probabilidad que se pide en el enunciado}

Independientemente del espacio muestral con el que se esté trabajando, se puede solicitar al estudiante el cálculo de distintos tipos de probabilidades; respecto a esta variable hemos considerado como categorías que se pida calcular la probabilidad simple, condicionada o compuesta, cada una de las cuales tiene diferente dificultad.

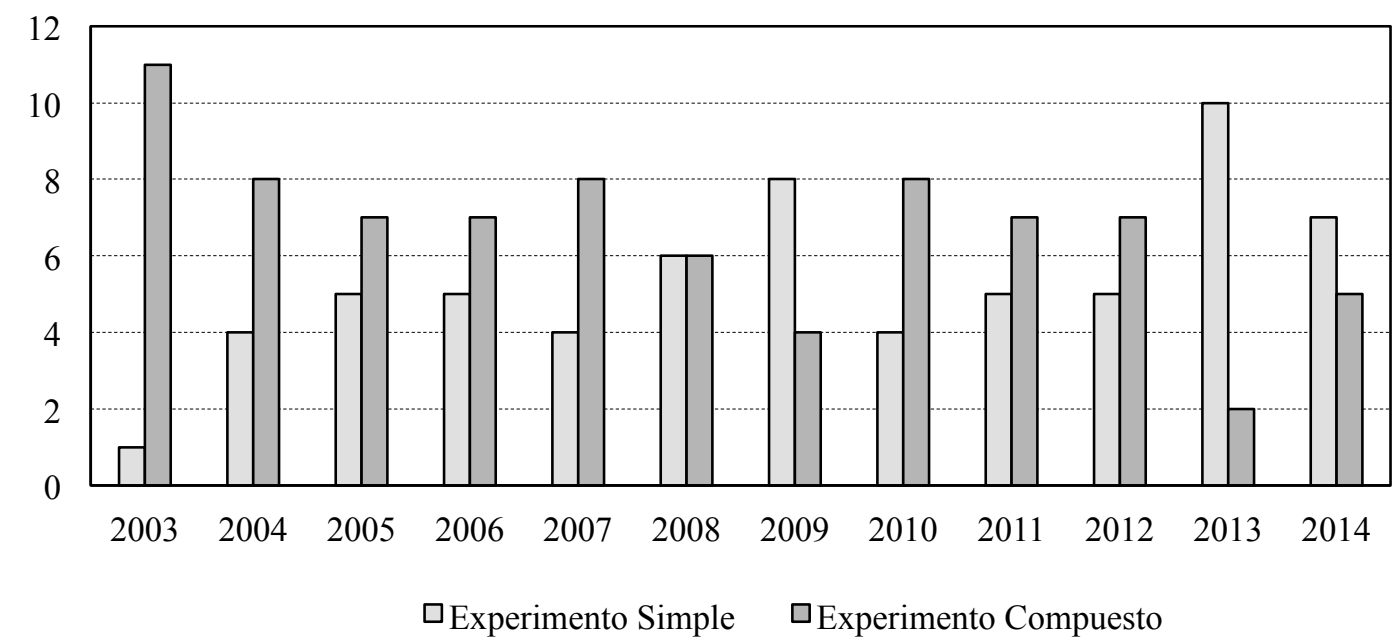

Figura 1. Tipo de experimento por año.

Hay que tener en cuenta que en un mismo problema pueden aparecer varias de estas probabilidades. Un ejemplo en el que observamos la inclusión de estas tres probabilidades lo tenemos en el ítem P3B, propuesto el año 2013, cuyo enunciado es el que sigue:

Ítem $P 3 B$ (2013). En una empresa el $65 \%$ de sus empleados habla inglés, de éstos, el $40 \%$ habla también alemán. De los que no hablan inglés, el $25 \%$ habla alemán. Se escoge un empleado al azar:

a. ¿Cuál es la probabilidad de que hable ambos idiomas?

b. ¿Cuál es la probabilidad de que hable alemán?

c. ¿Cuál es la probabilidad de que, sabiendo alemán, hable también inglés?

Analizando el ítem se observa que tanto la probabilidad simple como la probabilidad condicionada aparecen en el enunciado del ejercicio y en los apartados de resolución. De los datos proporcionados en el enunciado se tiene que "el $65 \%$ de sus empleados habla inglés" corresponde a una probabilidad simple y el resto de información va asociada a una probabilidad condicionada. Centrando la atención en cada uno de los apartados se concluye que: la resolución del apartado a) conlleva el cálculo de una probabilidad compuesta, ya que se pide la probabilidad de que un empleado hable ambos idiomas; por otro parte la probabilidad pedida en el apartado b) es de tipo simple debido a que hay que obtener la probabilidad de que un empleado hable alemán; por último, considerando que se especifica una condición y el estudiante ha de diferenciar entre la condición y el condicionado, el apartado c) corresponde a una probabilidad condicionada. 
La Figura 2 resume los resultados obtenidos al analizar la probabilidad que se solicita en los ítems, destacando por su frecuencia los problemas de probabilidad condicionada. Cabe destacar la importancia que tiene el dominar dicho concepto para el desarrollo y comprensión de muchos otros elementos de estadística (por ejemplo, correlación, regresión, nivel de significación de un contraste de hipótesis, etc., véase Falk, 1986). Además, se ha de señalar que la probabilidad condicionada puede ser empleada en la toma de decisiones de la vida cotidiana y profesional, donde se evalúan las consecuencias de dichas decisiones bajo diversos supuestos. Esta importancia puede incidir en el hecho de la frecuencia con que se pide calcularla en estos problemas.

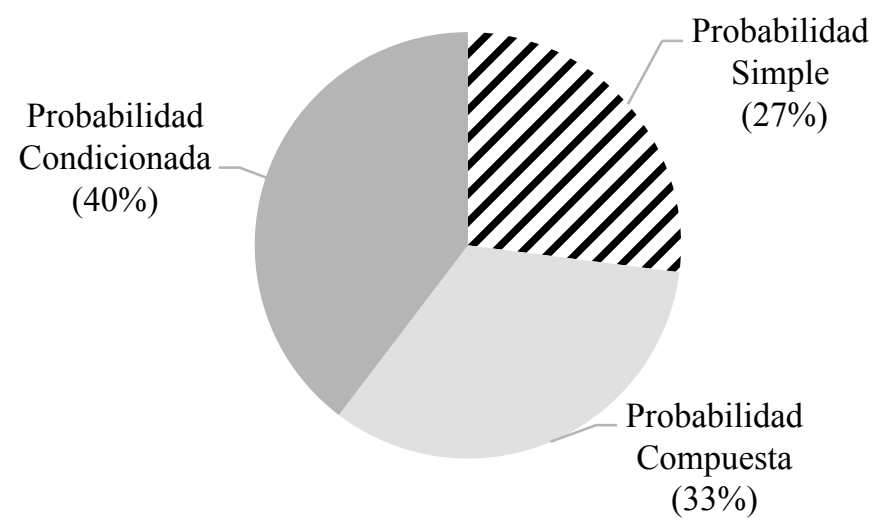

Figura 2. Clasificación en función al tipo de probabilidad que se pide.

Al estudiar la distribución de esta variable por año se observa que en muchos de ellos aparecen de forma conjunta las tres probabilidades, en el enunciado y/o en los apartados que deben ser resueltos, (véase Figura 3). De los 12 ítems propuestos cada año, la probabilidad condicional aparece más del 66\% (aproximadamente la tercera parte). Los otros dos tipos son más variable: la probabilidad simple a veces aparece sólo en 4 a 5 ítems (la mitad de los años) hasta llegar en 2003 a todos los problemas. La probabilidad compuesta llega el mismo año a 11 ítems, y entre 6 y 10 el resto de años.

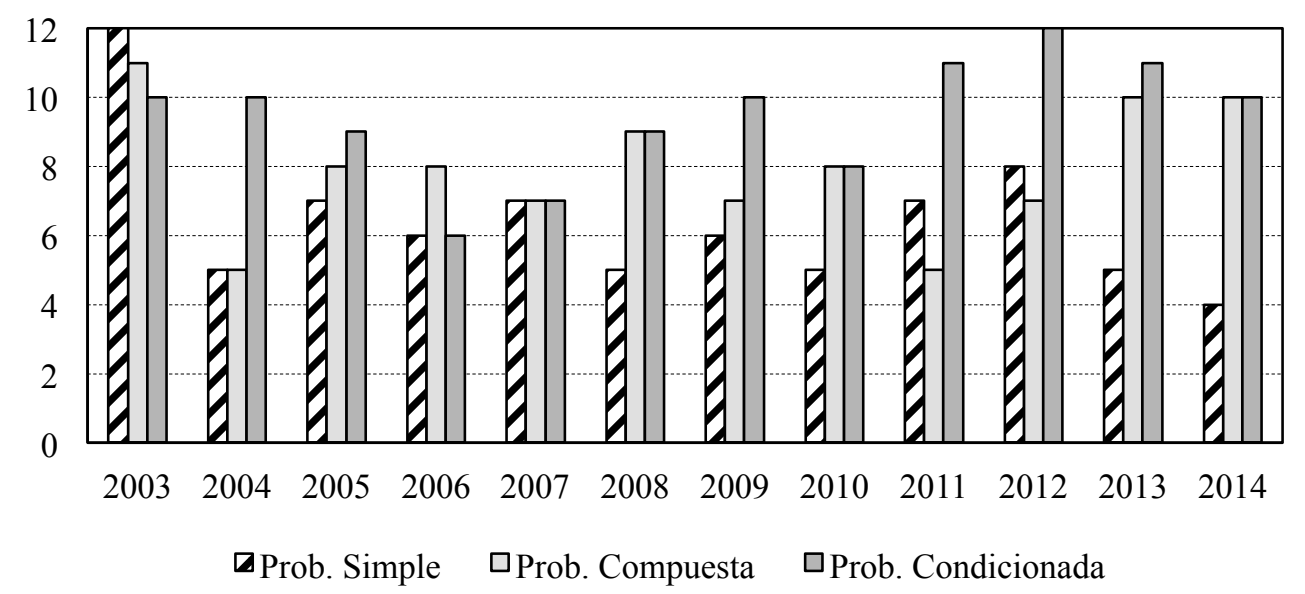

Figura 3. Clasificación de los ítems según periodo seleccionado y probabilidad que se pide. 
Del estudio realizado, se puede concluir que existe una alta tendencia a utilizar un único problema con el fin de evaluar la comprensión de dos o tres tipos de probabilidades y su discriminación por parte de los estudiantes, discriminación que es compleja, según se ha indicado en investigaciones previas (Díaz, 2007; Contreras, 2011).

\subsection{Teoremas o propiedades utilizadas}

A lo largo de esta sección se mostrarán los resultados que se han obtenido en el análisis de los teoremas (teorema de la probabilidad total y de Bayes) o propiedades que son necesarias para la resolución del problema.

La dificultad del empleo de los teoremas radica fundamentalmente en la detección de las dos particiones que sufre el espacio muestral junto con los sucesos y probabilidades que se describen con ellas. El hecho de no actuar de la forma adecuada puede provocar errores en la resolución del problema (véase Díaz, 2007). A modo de ejemplo se presenta la Figura 4 en la cual se observa que en un primer lugar se tiene la primera partición del espacio muestral formado por los sucesos $A_{1}$ y $A_{2}$ y posteriormente se ha realizado una segunda partición la cual conduciría a la obtención de probabilidades condicionales y compuestas (sucesos $B_{1}, B_{2}, B_{3}$ y $B_{4}$ ).

Tras el análisis de cada una de las pruebas se ha detectado que de los 144 ítems que forman la muestra aproximadamente 72 están relacionados con la aplicación de al menos uno de los teoremas. Este hecho añade dificultad a la prueba ya que generalmente la pregunta no es directa y el estudiante deberá identificar la aplicación de estos teoremas para la resolución del mismo.

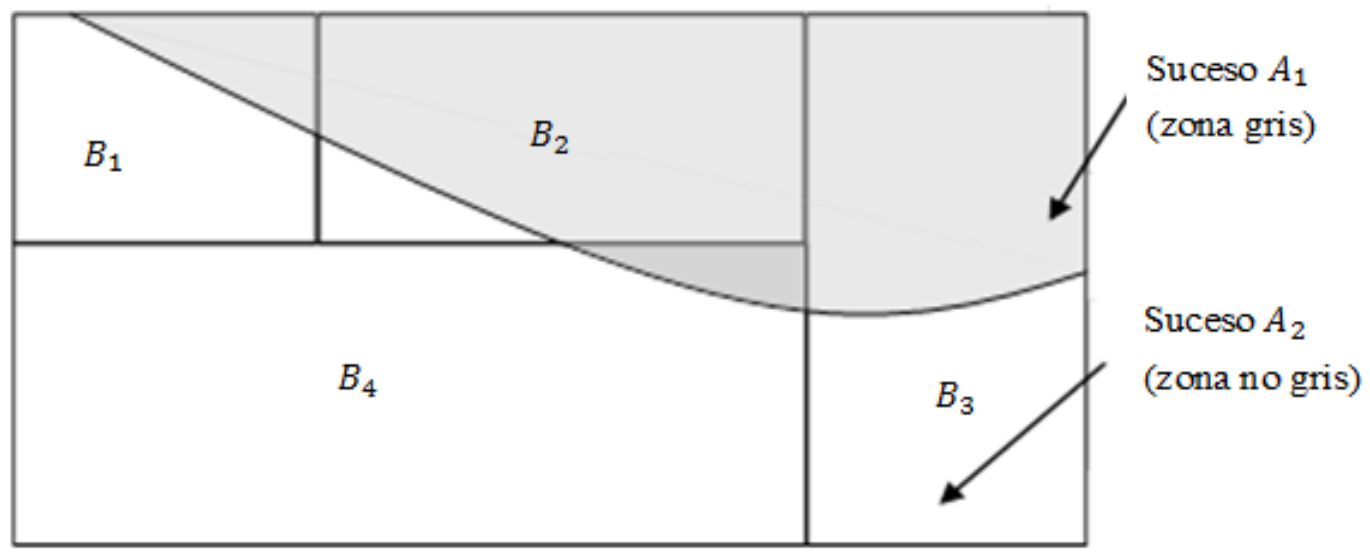

Figura 4. Partición del espacio muestral (teorema probabilidad total y de Bayes)

Un ejemplo de este tipo de ítems lo encontramos en la prueba 5, opción A, de 2010, donde se aprecia la necesidad de aplicar tanto el teorema de la probabilidad total como el teorema de Bayes:

Ítem P5A (2010). Un alumno va a la Facultad en autobús el $80 \%$ de los días y el resto en su coche. Cuando va en autobús llega tarde el $20 \%$ de las veces y cuando va en coche llega a tiempo sólo el $10 \%$ de las veces. Elegido un día cualquiera al azar, determine: 
a. La probabilidad de que llegue a tiempo a clase y haya ido en autobús.

b. La probabilidad de que llegue tarde a clase.

c. Si ha llegado a tiempo a clase, ¿cuál es la probabilidad de que no haya ido en autobús?

Para resolver el problema, en primer lugar interpretamos del enunciado que el espacio muestral está particionado con los sucesos $A=$ ir en autobús y $C=$ ir en coche, los cuales son sucesos complementarios entre sí. Por otro lado, se detecta que la segunda partición que se realiza sobre el mismo espacio muestral es la que describen los sucesos $T=$ llegar tarde a la facultad y su complementario, $\bar{T}$. Los datos que se tienen directamente de la información recogida en el enunciado son:

$$
P(A)=0^{\prime} 80 ; P(T \mid A)=0^{\prime} 20 \text { у } P(\bar{T} \mid C)=0^{\prime} 10 .
$$

En primer apartado el alumno deberá ver la equivalencia existente ente el suceso un alumno llegue a tiempo a clase y haya ido en autobús y la intersección entre los sucesos $T$ y $A$. Debido a la dependencia existente entre ambos sucesos se realizará el cálculo recurriendo a la expresión correspondiente a la probabilidad condicionada obteniendo que

$$
P(T \cap A)=P(T \mid A) \cdot P(A)=0^{\prime} 80 \cdot 0^{\prime} 80=0^{\prime} 64
$$

El enunciado del segundo apartado puede generar en el alumno cierta confusión ya que puede pensar que la probabilidad solicitada es de tipo simple. Sin embargo si deduce que la probabilidad que se solicita corresponde a uno de los sucesos obtenidos en la segunda partición (o subpartición) entonces recurrirá al uso del teorema de la probabilidad total para obtener la probabilidad de que llegue a tiempo independientemente de si va en autobús o en coche,

$$
\left(P(\bar{T})=P(A) \cdot P(\bar{T} \mid A)+P(C) \cdot P(\bar{T} \mid C)=0^{\prime} 8 \cdot 0^{\prime} 8+0^{\prime} 2 \cdot 0^{\prime} 10=0^{\prime} 66\right) .
$$

Por tanto, la probabilidad pedida de llegar tarde sería 1-0'66 = 0'34.

Finalmente, el tercer apartado pide calcular la probabilidad de que el alumno no haya ido en autobús sabiendo que no ha llegado tarde. Esta probabilidad, semejante a la probabilidad de ir en coche, sabiendo que no ha llegado tarde, se obtiene mediante el teorema de Bayes o probabilidad inversa, cuya expresión es:

$$
P(A \mid \bar{T})=\frac{P(A) \cdot P(\bar{T} \mid A)}{P(A) \cdot P(\bar{T} \mid A)+P(C) \cdot P(\bar{T} \mid C)} .
$$

Recordemos que el denominador el teorema de Bayes es exactamente la expresión correspondiente al teorema de la probabilidad total que ha sido empleada en el apartado anterior. Teniendo en cuenta los datos recogidos en el enunciado y el resultado obtenido en el segundo apartado se concluye que $P(A \mid \bar{T})=0^{\prime} 0 \hat{3}$.

En la Figura 5 se observa que en la mayoría de los ítems y en todos los años hay que aplicar el teorema de la probabilidad total y/o teorema de Bayes. El hecho de que en cinco de los años estudiados aparezcan el mismo número de ítems en los que se pide el cálculo de ambos teoremas no es llamativo ya que la aplicación del teorema de Bayes supone una aplicación previa del teorema de la probabilidad total. 


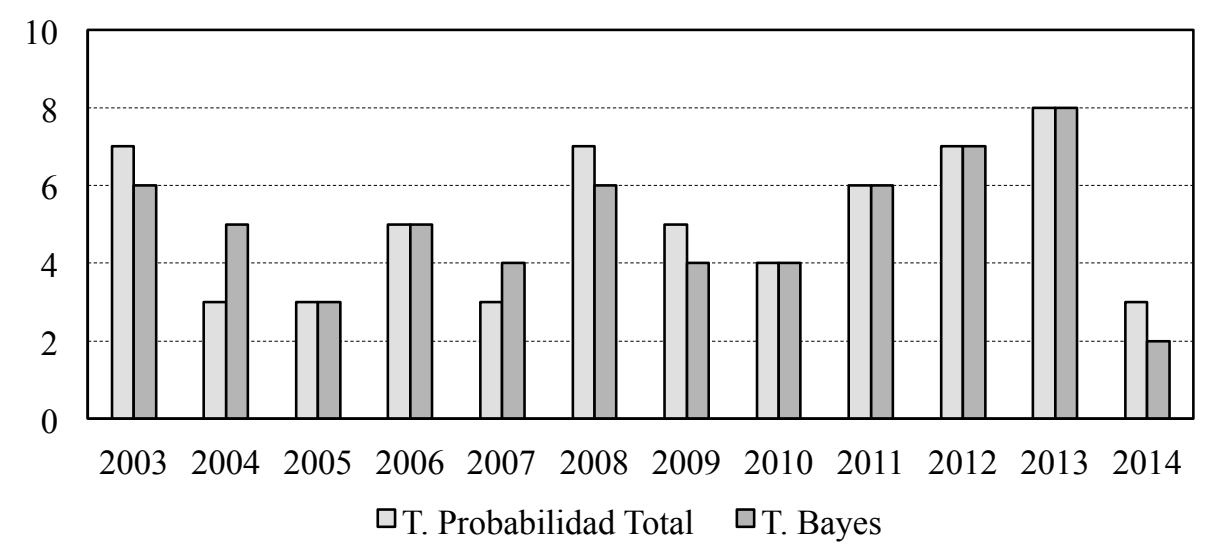

Figura 5. Clasificación según el teorema empleado.

El problema adquiere complejidad cuando en el ítem aparece la aplicación del teorema de Bayes sin haber solicitado anteriormente el cálculo del teorema de la probabilidad total (por ejemplo el año 2004). Esto corrobora la idea de que se pretende, con este tipo de problemas, añadir mayor dificultad al estudiante en la ejecución del ejercicio.

\subsection{Contexto}

En este último punto, se va analizar el contexto en el que son formulados los problemas propuestos. El principal objetivo del contexto en el que se desarrolla un problema es mostrar la unión existente entre los conceptos y las situaciones reales con el fin de darle sentido al aprendizaje de los contenidos matemáticos y conseguir de esta forma motivar el interés del estudiante por el aprendizaje de las matemáticas, haciéndoles ver la utilidad de dicha materia en diferentes contextos. En la clasificación de dicha variable se ha tenido en cuenta que los diferentes tipos de contextos son:

- Situación personal: Son las que están relacionadas con las actividades del día a día del alumno y tienen relevancia personal directa e inmediata para el estudiante. Incluyen referencias a actividades del alumno, su familia o amigos o el contexto escolar. Algunos ejemplos citados en el informe PISA son las compras, juegos, salud o transporte personal, deportes, viajes.

- Situación profesional: Son problemas que se centran en el mundo laboral, en situaciones adecuadas para los alumnos. Algunos ejemplos podrían ser problemas sobre medida o coste de un proceso de producción o una construcción, sobre diseño en carpintería, arquitectura o jardinería; coste o salario de mano de obra, etc. Este contexto es muy frecuente en la aplicación del teorema de Bayes.

- Situación Social: Serían problemas que el estudiante podría encontrar en su comunidad (comunidad de vecinos, ayuntamiento o ciudad, su país, etc.). Por ejemplo, se pueden incluir acá problemas relacionados con elecciones, transporte, demografía o publicidad. Incluimos también los juegos de azar.

- Situación Científica: Los problemas clasificados en la categoría científico hacen referencia a la aplicación de las matemáticas en ciencia y tecnología. Algunos ejemplos serían problemas relacionados con la meteorología, 
ecología, medicina, genética, o física. Esta situación es más abstracta que el resto, ya que implica la comprensión de un proceso tecnológico, una interpretación teórica o un problema matemático.

- Sin contexto: Son situaciones matemáticas abstractas en que no se incluye ninguna aplicación a la vida real, lo que contradice todas las recomendaciones sobre la enseñanza de la estadística.

La Tabla 2 recoge los contextos considerados en las pruebas PISA junto con un ejemplo de las pruebas PAU y la Figura 6 la distribución de contextos en los problemas.

Observamos que el contexto social ha sido el más aplicado, alcanzando un porcentaje del $38 \%$. Esta frecuencia es debida a que se ha incluido como parte de este contexto los juegos de azar.

Tabla 2. Ejemplos de problemas en los contextos recogidos en las pruebas PISA

\begin{tabular}{|c|c|}
\hline Contexto & $\begin{array}{c}\text { Ejemplo (ítems de PAU) } \\
\end{array}$ \\
\hline $\begin{array}{l}\text { Situación } \\
\text { personal: }\end{array}$ & $\begin{array}{l}\text { Ítem P6A (2008). Ana y Blas deciden jugar con un dado de la siguiente forma: Ana } \\
\text { lanza el dado y, si saca un } 6 \text {, gana y se acaba el juego. En caso contrario lanza Blas, } \\
\text { que gana si saca un } 2 \text { o un } 3 \text {, y también se acaba el juego. De no ocurrir esto, la partida } \\
\text { se acaba sin ganador. Halle la probabilidad de los siguientes sucesos: "gana Ana", } \\
\text { "gana Blas", "ninguno gana". }\end{array}$ \\
\hline $\begin{array}{c}\text { Situación } \\
\text { profesional: }\end{array}$ & $\begin{array}{l}\text { Ítem } P 6 B \text { (2008). En una industria de calzado se producen botas y sandalias. De cada } \\
12 \text { pares producidos, } 7 \text { pares son botas y } 5 \text { de sandalias. La probabilidad de que un par } \\
\text { de botas sea defectuoso es } 0 \text { '08 y de que lo sea un par de sandalias es } 0 \text { '03. Se escoge } \\
\text { al azar un par y resulta ser "no defectuoso". } \\
\text { a. ¿Cuál es la probabilidad de que se haya escogido un par de botas? } \\
\text { b. ¿Cuál es la probabilidad de que se haya escogido un par de sandalias? }\end{array}$ \\
\hline $\begin{array}{l}\text { Situación } \\
\text { Social: }\end{array}$ & $\begin{array}{l}\text { Item } P 4 A \text { (2013). En un Centro de Salud se ponen dos terapias, A y B, para dejar de } \\
\text { fumar. De las personas que acuden al Centro para dejar de fumar, el } 45 \% \text { elige la } \\
\text { terapia } \mathrm{A} \text {, y el resto la B. Después de un año el } 70 \% \text { de los que siguieron la terapia A y } \\
\text { el } 80 \% \text { de los que siguieron la B no han vuelto a fumar. Se elige al azar un usuario del } \\
\text { Centro que siguió una de las dos terapias. } \\
\text { a. Calcule la probabilidad de que un año después no haya vuelto a fumar. } \\
\text { b. Si transcurrido un año esa persona sigue sin fumar, calcule la probabilidad de } \\
\text { que hubiera seguido la terapia A. } \\
\text { c. Si transcurridos una año esa persona ha vuelto a fumar, calcule la } \\
\text { probabilidad de que hubiera seguido la terapia A. }\end{array}$ \\
\hline $\begin{array}{l}\text { Situación } \\
\text { Cientifica: }\end{array}$ & $\begin{array}{l}\text { Ítem P1A (2012). En un congreso de } 200 \text { jóvenes profesionales se pasa una encuesta } \\
\text { para conocer los hábitos en cuanto a contratar los viajes por internet. Se observa que } \\
120 \text { son hombres y que, de estos, } 84 \text { contratan los viajes por internet, mientras que } 24 \\
\text { de las mujeres no emplean esa vía. Elegido un congresista al azar, calcule la } \\
\text { probabilidad de que: } \\
\text { a. No contrate sus viajes por internet. } \\
\text { b. Use internet para contratar los viajes, si la persona elegida es una mujer. } \\
\text { c. Sea hombre, sabiendo que contrata sus viajes por internet. }\end{array}$ \\
\hline 年 & $\begin{array}{l}\text { Ítem } P 3 B(2013) \text {. Sean A y } \mathrm{B} \text { dos sucesos aleatorios independientes. Se sabe que } \\
P(A)=0{ }^{\prime} 3, P(B)=0 \text { ' } 4 \text {. Calcule las siguientes probabilidades: } \\
\quad \text { a. } \quad P(A \cap B) \text {. } \\
\text { b. } \quad P(A \mid B C) .\end{array}$ \\
\hline
\end{tabular}


Es de señalar la escasa presencia del contexto científico (sólo el 3\%) y en realidad no hemos encontrado problemas con contextos propiamente científicos entre los analizados; aunque algunos problemas se refieren a enfermedades y tratamientos, los hemos clasificado como contexto social porque se refiere al contexto social próximo del alumno (por ejemplo, un Centro de Salud), pero no presenta conceptos científicos que el estudiante haya de interpretar para resolver el problema.

Es también muy alto el porcentaje de problemas que carecen de contexto, lo que contradice todas las recomendaciones sobre la enseñanza de la estadística. En estos casos el estudiante no puede comprender el interés de aplicación de las matemáticas, pues no se especifica el objetivo del cálculo de las probabilidades pedidas. La finalidad que se persigue con este tipo de problemas es simplemente que el alumno aplique reglas matemáticas de cálculo sin objetivo concreto y comprobar el reconocimiento de la simbología utilizada dentro del ámbito de la probabilidad. Sin embargo, cabe señalar que el $20 \%$ de los ítems propuestos en las pruebas PAU tienen este carácter abstracto (véase Figura 6).

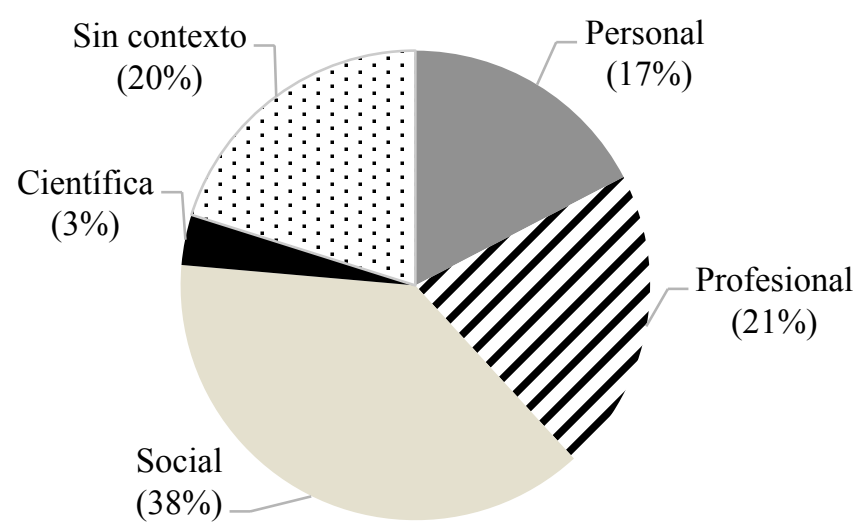

Figura 6. Clasificación de los ítems según el contexto

Al analizar los contextos por año (Figura 7), observamos gran variabilidad; aunque el contexto social (incluidos juegos de azar) es predominante. En algunos años la mayor frecuencia es de contextos profesionales, donde el alumno podrá apreciar mejor la aplicación de la probabilidad en otras materias y actividades.

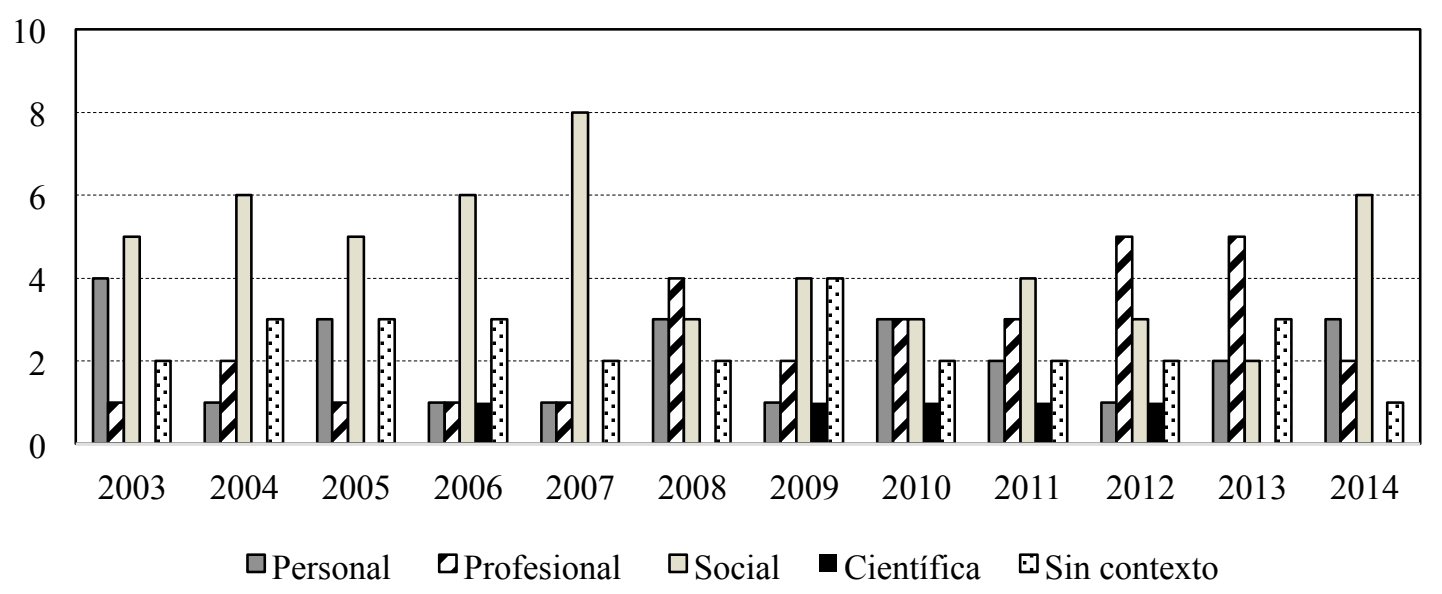


Figura 7. Contextos de los ítems por cada año

\section{Conclusiones}

Las pruebas analizadas incluyen un problema de probabilidad, más concretamente de cálculo de probabilidades, que, generalmente pide la probabilidad compuesta y condicional. Como hemos indicado, posiblemente la importancia del concepto de probabilidad condicional en la vida cotidiana y profesional incide en la frecuencia con que se proponen este tipo de ejercicios.

Sin embargo, basándonos en investigaciones previas sobre las dificultades de los estudiantes al resolver problemas de probabilidad, podemos deducir una alta complejidad de los problemas propuestos en las PAU sobre probabilidad. Por ejemplo, Díaz y de la Fuente $(2005 ; 2007)$ indican que al trabajar con probabilidad condicional los alumnos puede dar soluciones incorrectas cuando se invierte el orden temporal en que ocurren los sucesos condición y condicionado. Esto ocurre en todos los problemas en que el alumno ha de aplicar el teorema de Bayes. Igualmente muchos estudiantes confunden la probabilidad condicional y conjunta (Pollatsek, Well, Konold \& Hardiman, 1987; Ojeda, 1995) debido a que el lenguaje ordinario usado en los enunciados es poco riguroso. Por ello, pueden dar soluciones incorrectas en los apartados de los problemas en que se piden este tipo de probabilidad.

Es también habitual en los problemas de las PAU en Andalucía el uso de experimentos compuestos (muchas veces dependientes entre sí). Los alumnos podrían tener dificultad en identificar la dependencia o independencia de los experimentos, como se encontró en la investigación de Contreras (2011) en que los participantes debido a esta confusión utilizan fórmulas incorrectas en el cálculo de la probabilidad compuesta.

Muchos problemas analizados requieren la aplicación de uno de los teoremas de la probabilidad total o de Bayes o los dos simultáneamente. De acuerdo a Contreras (2011) y Totohasina (1992), la solución de estos problemas requiere, en primer lugar identificar las probabilidades de los sucesos simples dados en el enunciado, e interpretar la pregunta del enunciado en términos de probabilidad condicional. Seguidamente, se ha de efectuar una partición en el espacio muestral, aplicar la fórmula de la probabilidad compuesta en pasos sucesivos, para finalmente hallar la probabilidad inversa. Al analizar esta variable, se han mostrado estos pasos mediante la solución de un ejemplo, indicando también los puntos potencialmente difíciles en cada uno de los pasos, donde los autores citados indican posibles errores, que podrían cometer los alumnos en los problemas propuestos en las PAU.

De los resultados obtenidos se ha observado un porcentaje aproximado del $20 \%$ de ejercicios descontextualizados, algo menor que en la investigación de Caraballo (2010), en la que sólo el $64 \%$ de ejercicios contenía un contexto significativo. Llamamos la atención, no obstante, sobre la importancia del contexto, indicada en el modelo PISA (OCDE, 2009), ya que cuando un estudiante se mueve entre el contexto real del problema y el modelo matemático necesario para resolverlo, está matematizando y además permite apreciar las aplicaciones de la probabilidad.

En resumen, nuestro análisis indica una alta dificultad de los problemas propuestos de probabilidad en las pruebas de acceso, que debería ser tenida en cuenta por los diseñadores de las mismas en las sucesivas ediciones o en pruebas de evaluación alternativas que se propongan en el futuro. Aunque en nuestros antecedentes hemos 
encontrado análisis de otras pruebas de evaluación, ninguna se ha centrado en los problemas de probabilidad en las PAU. Por tanto en este estudio hemos pretendido completar la investigación previa realizando dicho análisis.

Finalmente queremos resaltar que aunque el contenido del currículo, incluye otros muchos temas de estadística (como variable aleatoria, distribuciones binomial y normal, correlación e inferencia) nuestro análisis muestra que las pruebas dan mucha mayor importancia al cálculo de la probabilidad condicional y conjunta que al resto de la estadística y probabilidad.

Agradecimientos. Proyecto EDU2013-41141-P (MEC) y grupo FQM126 (Junta de Andalucía).

\section{Referencias}

Arteaga, P. (2011). Evaluación de conocimientos sobre gráficos estadísticos y conocimientos didácticos de futuros profesores. Tesis Doctoral. Universidad de Granada, Granada.

Batanero, C., Arteaga, P., \& Gea, M. (2011). El currículo de estadística: Reflexiones desde una perspectiva internacional. UNO. Revista de Didáctica de las Matemáticas, 59, 9-17

Batanero, C., Gea, M., Arteaga, P., \& Contreras, J.M. (2014). La estadística en la educación obligatoria: Análisis del currículo español. Revista digital Matemática, Educación e Internet 14 (2). Disponible en: http://www.tec-digital.itcr.ac.cr/revistamatematica/.

Bisquerra, R. (1989). Métodos de investigación educativa. Barcelona: P.P.U.

Caraballo, R. (2010). Análisis de los items de las pruebas de evaluación de diagnóstico en competencia matemática para el segundo curso de la educación secundaria obligatoria en España, 2008-2009: un estudio exploratorio. Trabajo fin de Máster. Universidad de Granada, Granada.

Castellanos, M. T. (2013). Tablas y gráficos estadísticos en las pruebas Saber Colombia. (Trabajo fin de Máster no publicado). Universidad de Granada, Granada.

Contreras, J. M. (2011). Evaluación de conocimientos y recursos didácticos en la formación de profesores sobre probabilidad condicional. (Tesis Doctoral no publicada). Universidad de Granada, Granada.

Consejería de Educación. Junta de Andalucía (2008). ORDEN de 5 de agosto de 2008, por la que se desarrolla el currículo correspondiente al Bachillerato en Andalucía. Sevilla: Autor.

Díaz, C. (2007). Viabilidad de la enseñanza de la inferencia bayesiana en el análisis de datos en psicología. (Tesis doctoral no publicada). Universidad de Granada, Granada.

Díaz, C., \& de la Fuente, I. (2007). Validación de un cuestionario de razonamiento probabilístico condicional. REMA, 12(1). Disponible en: www.psico.uniovi.es/REMA/v12n1/a1/.

Díaz, C., \& de la Fuente, I. (2005). Razonamiento sobre probabilidad condicional e implicaciones para la enseñanza de la estadística. Epsilon, 59, 245-260.

Díaz, C., Ortiz, J. J., \& Serrano, L. (2008). Dificultades de los estudiantes de psicología en el cálculo de probabilidades inversas mediante el teorema de Bayes. Publicaciones, 35, 109123. 
Falk, R. (1986). Conditional probabilities: insights and difficulties. En R. Davidson, \& J. Swift (Eds.), Proceedings of the Second International Conference on Teaching Statistics. (pp. 292-297). Victoria, Canada: International Statistical Institute.

Godino, J. D. (1996). Mathematical concepts, their meanings and understanding. En L. Puig, \& A. Gutiérrez (Eds.), Proceedings of the 20th PME Conference (v.2, pp. 417-424). Universidad de Valencia.

Godino, J. D., Batanero, C., \& Font, V. (2007). The onto-semiotic approach to research in mathematics education. ZDM. The International Journal on Mathematics Education, 39 (1-2), 27-135.

Krippendorff, K. (1997). Metodología de análisis de contenido. Teoría y práctica. Barcelona: Paidos.

Mingorance, C. (2014). La estadística en las pruebas de diagnóstico andaluzas. Trabajo fin de Grado. Universidad de Granada, Granada.

MCD, Ministerio de Educación, Cultura y Deporte (2013). PISA 2012. Programa para la evaluación internacional de los alumnos. Informe español. Vol.1. Resultados y contexto. Madrid: Autor.

ME, Ministerio de Educación (2009). PISA 2009. Programa para la evaluación internacional de alumnos de la OCDE. Informe español. Madrid: Autor.

MEC, Ministerio de Educación y Ciencia (2007). Real Decreto 1467/2007, de 2 de noviembre, por el que se establece la estructura del Bachillerato y se fijan sus enseñanzas mínimas. Madrid: Autor.

MECD, Ministerio de Educación, Cultura y Deporte (2015). Real Decreto 1105/2014, de 26 de diciembre, por el que se establece el currículo básico de la Educación Secundaria Obligatoria y del Bachillerato. Madrid: Autor.

MP, Ministerio de la Presidencia (2008). Real Decreto 1892/2008, de 14 de noviembre, por el que se regula las condiciones para el acceso a las enseñanzas universitarias oficiales de grado y los procedimientos de admisión a las universidades públicas españolas. Madrid: Autor.

OCDE, Organización para la Cooperación y el Desarrollo Económico (2009). PISA 2009 assessment framework - key competencies in reading, mathematics and science. Paris: OCDE

Ojeda, A. M. (1995). Dificultades del alumnado respecto a la probabilidad condicional. UNO. Revista de Didáctica de las Matemáticas, 5,37-55.

Ortiz, J. J. (2002). Significado de los conceptos probabilísticos elementales en los textos de Bachillerato. Tesis Doctoral. Universidad de Granada, Granada.

Pollatsek, A., Well, A. D., Konold, C. \& Hardiman, P. (1987). Understanding conditional probabilities. Organitation, Behavior and Human Decision Processes, 40, 255-269.

Rico, L. (2006). Marco teórico de evaluación en PISA sobre matemáticas y resolución de problemas. Revista de Educación, (Extraordinario 2006), 275-294.

Totohasina, A. (1992). Méthode implicative en analyse de données et application á l'analyse de conceptions d'étudiants sur la notion de probabilité conditionnelle. (Tesis Doctoral no publicada). Universidad Rennes I.

\section{Referencias a los autores}

María del Mar López-Martín, Universidad de Granada (España)

mariadelmarlopez@ugr.es 
José Miguel Contreras, Universidad de Granada (España), jmcontreras@ugr.es Magdalena Carretero, IES Miguel de Cervantes, Granada (España) magdasof72@hotmail.com

Luis Serrano, Universidad de Granada (España), 1serrano@ugr.es 


\title{
Analysis of probability problems proposed in the entrance to university tests in Andalucía
}

\author{
María del Mar López-Martín, Universidad de Granada (España) \\ José Miguel Contreras, Universidad de Granada (España \\ Magdalena Carretero, IES Miguel de Cervantes, Granada (España) \\ Luis Serrano, Universidad de Granada (España)
}

In this work we analyse the contents of the probability problems proposed for the university entrance tests in Andalucía (Spain). Specifically, we examine the problems included to the students who selected the specialty of Social Sciences.

The pertinence of this project is clear, since these tests often influence (more than the curricular guidelines) what is taught to these students, and our results may help the teachers to prepare their students for these tests. These preliminary results consist in the analysis of all the problems posed from 2003 to 2014 (144 problems in total).

We base on the onto-semiotic approach to mathematics education (Godino, Batanero, \& Font, 2007), where meaning is modelled in terms of systems of the practices carried out when solving a given problem. In these practices different type of objects intervene; "object"e is understood in a broad sense and includes problemsituations; procedures; concepts and properties. This analysis allows to identify the correspondence between assessment and curricular guidelines and to show the complex work required from the student.

In our study, we focus only on the probability content of this curriculum. Each test includes a specific problem related to this content. Moreover, as we will show in our analysis this problem is centre specifically in "Reinforcing ideas about prior and posterior probabilities, joint probability, conditional probability; total probability and the Bayes' rule".

Our analysis reveals the difficulty of the probability problems included to the students in the university entrance tests. The scores obtained by the students in these tests often determine that they can enter their preferred career. Then, our results should be taken into account by the test designers in order to build more reasonable assessment tests in the future. 